\title{
A MATHEMATICAL APPROACH TO JARZYNSKI'S IDENTITY IN NONEQUILIBRIUM STATISTICAL MECHANICS
}

\author{
EVELINA SHAMAROVA \\ Grupo de Física-Matemática da Universidade de Lisboa \\ Portugal \\ evelina@cii.fc.ul.pt
}

Received 18 April 2008

Communicated by F. Fagnola

\begin{abstract}
We develop a mathematical approach to the nonequilibrium work theorem which is traditionally referred to in statistical mechanics as Jarzynski's identity. We suggest a mathematically rigorous formulation and proof of the identity.
\end{abstract}

Keywords: Nonequilibrium work theorem; Jarzynski's identity; nonequilibrium statistical mechanics; probability measures on phase space paths.

AMS Subject Classification: 60J25, 60J35, 82C05

\section{Introduction}

The nonequilibrium work theorem is an equation in statistical mechanics that relates the free energy difference $\Delta F$ to the work $W$ carried out on a system during a nonequilibrium transformation. The identity appeared in different, but as we show below, equivalent formulations in Refs. 1 and 2 in 1997, and in the series of papers, Refs. 4-7 in 1977-1981.

In physics literature, the identity is usually written in the form

$$
\left\langle e^{-\beta W}\right\rangle=e^{-\beta \Delta F}
$$

where the average is taken over all possible system trajectories in the phase space, and $\beta$ is an inverse temperature. The identity first appeared in this form in Refs. 1 and 2. Traditional equilibrium thermodynamics tells us that $\langle W\rangle \geq \Delta F$ while the transformation of the system is infinitely slow. The identity (1) is a stronger statement, and in addition to this, it is valid for arbitrary transformations of the system. The identity is used effectively in computer simulations, as well as in experimental physics, to calculate the free energy difference between two states of the system by running many trajectories and taking the average value of $e^{-\beta W}$ (see Refs. 8-12, 14-16 and references therein). 
The paper Ref. 3, discusses the connection between two different versions of the identity, and shows that Refs. 4-7 use a different definition of work. The identity obtained in Refs. 4-7 (referred to below as Bochkov-Kuzovlev's identity) reads:

$$
\left\langle e^{-\beta W_{0}}\right\rangle=1
$$

where $W_{0}$ is the work (in Bochkov-Kuzovlev's sense) performed on the system, and the angle brackets have the same meaning as in (1). The present paper shows that Jarzynski's and Bochkov-Kuzovlev's identities easily follow from each other.

Since the identities involve taking an "average over trajectories", it is natural to interpret this average as the expectation relative to a probability measure on trajectories, while assuming that the system evolves stochastically. In terms of expectations, the identities can be represented by the formulas

$$
\mathbb{E}\left[e^{-\beta W}\right]=e^{-\beta \Delta F} \text { and } \quad \mathbb{E}\left[e^{-\beta W_{0}}\right]=1,
$$

where $\mathbb{E}$ is the expectation relative to a probability measure on phase space paths. For this probability measure, some analytical assumptions under which the identities hold are found.

\section{Notation and Assumptions}

Let us assume that the evolution of our system is described by a Markov process $\Gamma_{t}(\omega), t \in[0, T]$, given through its transition density function. Let $X=\mathbb{R}^{2 d}$ be the phase space for our system, i.e. the set of values of $\Gamma_{t}$. We assume that at time $t=0$ the distribution on the phase space $X$ is given by the following density function:

$$
q_{\lambda_{0}}(x)=\frac{e^{-\beta \mathcal{H}\left(x, \lambda_{0}\right)}}{\int_{X} e^{-\beta \mathcal{H}\left(x^{\prime}, \lambda_{0}\right)} d x^{\prime}}=\frac{1}{Z_{\lambda_{0}}} e^{-\beta \mathcal{H}\left(x, \lambda_{0}\right)},
$$

where $\mathcal{H}(\cdot, \lambda): X \rightarrow \mathbb{R}$ is a Hamiltonian parametrized by an externally controlled parameter $\lambda \in \Lambda, \Lambda \subset \mathbb{R}^{l}$ is an open set; $\beta=\frac{1}{k_{\mathrm{B}} T}, k_{\mathrm{B}}$ is the Boltzmann constant, $T$ is the temperature of the system, $Z_{\lambda_{0}}=\int_{X} e^{-\beta \mathcal{H}\left(x, \lambda_{0}\right)} d x$ is the partition function. We assume that for all $\lambda_{0} \in \Lambda, \int_{X} e^{-\beta \mathcal{H}\left(x, \lambda_{0}\right)} d x<\infty$. We consider the situation when the external parameter $\lambda$ is a function of time $[0, T] \rightarrow \Lambda$, i.e. we actually consider a time-dependent Hamiltonian $\mathcal{H}(x, \lambda(t))$. Let $\mathbb{E}_{\lambda_{0}}$ denote the expectation relative to the measure $q_{\lambda_{0}}(x) d x$. Below we assume that the changing in time external parameter $\lambda$ belongs to the space

$$
V[0, T]=\left\{\lambda:[0, T] \rightarrow \Lambda, \lambda=\lambda_{\mathrm{c}}+\lambda_{\text {step }} ; \lambda_{\mathrm{c}} \in \mathrm{C}^{V}, \lambda_{\text {step }} \in \mathcal{L}_{\text {step }}\right\},
$$

where $\mathrm{C}^{V}=\mathrm{C}^{V}[0, T]$ is the space continuous function of bounded variation on $[0, T]$, and

$$
\mathcal{L}_{\text {step }}=\mathcal{L}_{\text {step }}[0, T]=\left\{\lambda(\cdot)=\sum_{i=0}^{n-1} \lambda_{i} \mathbb{I}_{\left[t_{i}, t_{i+1}\right)}(\cdot)+\lambda_{n} \mathbb{I}_{\{T\}}(\cdot)\right\}
$$

is the space of right continuous step functions corresponding to different partitions $\mathcal{P}=\left\{0=t_{0}<\cdots<t_{n}=T\right\}$ of $[0, T]$ and different finite sets of values $\left\{\lambda_{i}\right\}$. 
To emphasize the fact that the function $\lambda \in V[0, T]$ gives rise to the process $\Gamma_{t}$, we will use the notation $\Gamma_{t}^{\lambda}$. Let $p_{\lambda}(s, x, t, y), s, t \in[0, T], s<t, x, y \in X$, be the transition density function for $\Gamma_{t}^{\lambda}$. Let $X^{[0, T]}$ denote the space of all paths $[0, T] \rightarrow X$. In terms of $p_{\lambda}$ we can construct a probability measure $\mathbb{L}_{\lambda}$ on $X^{[0, T]}$ by means of the finite-dimensional distributions

$$
\begin{aligned}
\int_{X^{[0, T]}} & f\left(\omega\left(t_{0}\right), \omega\left(t_{1}\right), \ldots, \omega\left(t_{n}\right)\right) \mathbb{L}_{\lambda}(d \omega) \\
= & \int_{X} d x_{0} q_{\lambda\left(t_{0}\right)}\left(x_{0}\right) \int_{X} d x_{1} p_{\lambda}\left(t_{0}, x_{0}, t_{1}, x_{1}\right) \ldots \\
& \cdot \int_{X} d x_{n} p_{\lambda}\left(t_{n-1}, x_{n-1}, t_{n}, x_{n}\right) f\left(x_{0}, x_{1}, \ldots, x_{n}\right),
\end{aligned}
$$

where $\left\{0=t_{0}<\cdots<t_{n}=T\right\}$ is a partition of the interval $[0, T]$, and $f: X^{n+1} \rightarrow \mathbb{R}$ is a bounded and measurable function. By Kolmogorov's extension theorem, the right-hand side of this equality defines a probability measure on the minimal $\sigma$ algebra of $X^{[0, T]}$ generated by all cylindrical sets. We denote this $\sigma$-algebra by $\sigma_{\mathrm{c}}\left(X^{[0, T]}\right)$.

Assumption 1. The measure $\mathbb{L}_{\lambda}$ is concentrated on the right continuous trajectories without discontinuities of the second kind.

Assumption 1 is fulfilled, for example, when the function $p_{\lambda}$ satisfies one of the conditions given in Ref. 17 (Chap. 2, paragraph 1). Also, we assume that the $\sigma$-algebra $\sigma_{\mathrm{c}}\left(X^{[0, T]}\right)$ is augmented with all subsets of $\mathbb{L}_{\lambda}$-null sets.

We take $X^{[0, T]}$ as the probability space, i.e. we set $\Omega=X^{[0, T]}$, and $\mathbb{L}_{\lambda}$ as the probability measure on it. Then, $\mathbb{L}_{\lambda}$-a.s., $\Gamma_{t}^{\lambda}(\omega)=\omega(t)$.

For each fixed $\bar{\lambda} \in \Lambda$, we introduce another transition density function $p(s, x, t, y, \bar{\lambda})$ which represents the situation when the system evolves being controlled by a constant in time parameter. The following Assumption 2 is the key assumption under which the nonequilibrium work theorem holds.

Assumption 2. If $\left.\lambda\right|_{[s, t]} \equiv \bar{\lambda}$, then $p_{\lambda}(s, x, t, y)=p(s, x, t, y, \bar{\lambda})$, where $p(s, x, t, y, \bar{\lambda})$ conserves the canonical distribution on the phase space $X$. Specifically, it satisfies the identity

$$
\int_{X} q_{\bar{\lambda}}(x) p(s, x, t, y, \bar{\lambda}) d x=q_{\bar{\lambda}}(y) .
$$

We make further assumptions:

Assumption 3. If $\lambda$ is constant on $[s, t)$, and discontinuous at the point $t$, then for Lebesgue almost all $x \in X$,

$$
\int_{X} d y p_{\lambda}(s, x, t, y) f(y)=\int_{X} d y p(s, x, t, y, \lambda(s)) f(y),
$$

where $f: X \rightarrow \mathbb{R}$ is bounded and Borel measurable. 
Assumption 4. For all $t \in[0, T)$, and for all compacts $K \subset X$,

$$
\lim _{\delta \rightarrow 0+} \sup _{x \in K}\left[\int_{X} p(t, x, t+\delta, y, \lambda(t)) f(y) d y-f(x)\right]=0,
$$

where $f: X \rightarrow \mathbb{R}$ is bounded and continuous.

Lemma 1. Let $\mathcal{P}=\left\{0=t_{0}<t_{1}<\cdots<t_{n}=T\right\}$ be a partition of $[0, T]$, and let $\lambda(\cdot)=\sum_{i=0}^{n-1} \lambda_{i} \mathbb{I}_{\left[t_{i}, t_{i+1}\right)}(\cdot)+\lambda_{n} \mathbb{I}_{\{T\}}(\cdot)$. We assume that if $\left.\lambda\right|_{[s, t]} \equiv \bar{\lambda}$, where $\bar{\lambda} \in \Lambda$ is constant, then $p_{\lambda}(s, x, t, y)=p(s, x, t, y, \bar{\lambda})$. Further we assume that the function $p_{\lambda}$ satisfies Assumption 3. Then, for all $s, t, 0 \leq s<t \leq T$, for all $x \in X$, and for all bounded and Borel measurable functions $f: X \rightarrow \mathbb{R}$,

$$
\begin{aligned}
\int_{X} d y & p_{\lambda}(s, x, t, y) f(y) \\
= & \int_{X} d x_{1} p\left(s, x, \tau_{1}, x_{1}, \lambda(s)\right) \int_{X} d x_{2} p\left(\tau_{1}, x_{1}, \tau_{2}, x_{2}, \lambda\left(\tau_{1}\right)\right) \cdots \\
& \cdot \int_{X} d y p\left(\tau_{k}, x_{k}, t, y, \lambda\left(\tau_{k}\right)\right) f(y),
\end{aligned}
$$

where $\left\{s<\tau_{1}<\cdots<\tau_{k}<t\right\}=(\mathcal{P} \cup\{s, t\}) \cap[s, t]$.

Proof. The proof follows from Assumption 3.

\section{Jarzynski's Identity}

Let $\lambda=\lambda_{\mathrm{c}}+\lambda_{\text {step }}$ be the decomposition of $\lambda \in V[0, T]$ into a sum of a $\lambda_{\mathrm{c}} \in \mathrm{C}^{V}[0, T]$ and a $\lambda_{\text {step }} \in \mathcal{L}_{\text {step }}[0, T]$. Further let $\partial_{\lambda} \mathcal{H}: X \times \Lambda \rightarrow \mathbb{R}^{l}$ denote the partial derivative with respect to the second argument (i.e. with respect to the control parameter). We assume that the partial derivative $\partial_{\lambda} \mathcal{H}$ exists on $X \times \lambda([0, T])$, and is bounded. Also, we assume that for each fixed $\bar{\lambda} \in \lambda([0, T]), \mathcal{H}(\cdot, \bar{\lambda})$ is bounded and Borel measurable. Everywhere below, the probability space $\Omega$ is the space $X^{[0, T]}$. We define the work $W_{\lambda}: \Omega \rightarrow \mathbb{R}$ performed on the system by the formula

$$
\begin{aligned}
W_{\lambda}(\omega)= & \int_{0}^{T}\left\langle\partial_{\lambda} \mathcal{H}\left(\Gamma_{t}^{\lambda}(\omega), \lambda(t)\right), d \lambda_{\mathrm{c}}(t)\right\rangle_{\mathbb{R}^{l}} \\
& +\sum_{i=1}^{n}\left(\mathcal{H}\left(\Gamma_{t_{i}}^{\lambda}(\omega), \lambda\left(t_{i}\right)\right)-\mathcal{H}\left(\Gamma_{t_{i}}^{\lambda}(\omega), \lambda\left(t_{i}-0\right)\right)\right),
\end{aligned}
$$

where $\left\{0=t_{0}<t_{1}<\cdots<t_{n}=T\right\}$ are discontinuity points of $\lambda,\langle,\rangle_{\mathbb{R}^{l}}$ is the scalar product in $\mathbb{R}^{l} \supset \Lambda$, and the integral on the right-hand side is the LebesgueStieltjes integral, i.e. the sum of the Lebesgue-Stieltjes integrals with respect to the components of $\lambda_{c}$. In the following, we skip the sign of the scalar product in the 
first term of (5), and simply write

$$
\begin{aligned}
W_{\lambda}(\omega)= & \int_{0}^{T} \partial_{\lambda} \mathcal{H}\left(\Gamma_{t}^{\lambda}(\omega), \lambda(t)\right) d \lambda_{\mathrm{c}}(t) \\
& +\sum_{i=1}^{n}\left(\mathcal{H}\left(\Gamma_{t_{i}}^{\lambda}(\omega), \lambda\left(t_{i}\right)\right)-\mathcal{H}\left(\Gamma_{t_{i}}^{\lambda}(\omega), \lambda\left(t_{i}-0\right)\right)\right) .
\end{aligned}
$$

Let $F_{\lambda}=-\frac{1}{\beta} \ln Z_{\lambda}$ (free energy of the system), and let $\Delta F=F_{\lambda(T)}-F_{\lambda(0)}$ (free energy difference). Let $\mathbb{E}_{\mathbb{L}_{\lambda}}$ denote the expectation relative to the measure $\mathbb{L}_{\lambda}$.

\subsection{Jarzynski's identity, case $\lambda \in \mathcal{L}_{\text {step }}[0, T]$}

Clearly, if $\lambda=\sum_{i=0}^{n-1} \lambda_{i} \mathbb{I}_{\left[t_{i}, t_{i+1}\right)}+\lambda_{n} \mathbb{I}_{\{T\}} \in \mathcal{L}_{\text {step }}[0, T]$,

$$
W_{\lambda}(\omega)=\sum_{i=1}^{n}\left(\mathcal{H}\left(\Gamma_{t_{i}}^{\lambda}(\omega), \lambda_{i}\right)-\mathcal{H}\left(\Gamma_{t_{i}}^{\lambda}(\omega), \lambda_{i-1}\right)\right) .
$$

Theorem 1. (Jarzynski's identity: case $\left.\lambda \in \mathcal{L}_{\text {step }}[0, T]\right)$ Let $\lambda \in \mathcal{L}_{\text {step }}[0, T]$, and let the transition density function $p_{\lambda}$ satisfy Assumptions 2 and 3 . Further let $\mathcal{H}(\cdot, \bar{\lambda})$ be bounded and Borel measurable on $X$ for each fixed $\bar{\lambda} \in \lambda([0, T])$. Then the function $e^{-\beta W_{\lambda}}$ is $\mathbb{L}_{\lambda}$-integrable, and

$$
\mathbb{E}_{\mathbb{L}_{\lambda}}\left[e^{-\beta W_{\lambda}}\right]=e^{-\beta \Delta F} .
$$

Proof. $\mathbb{L}_{\lambda \text {-a.s., }}$

$$
W_{\lambda}(\omega)=\sum_{i=1}^{n}\left(\mathcal{H}\left(\omega\left(t_{i}\right), \lambda_{i}\right)-\mathcal{H}\left(\omega\left(t_{i}\right), \lambda_{i-1}\right)\right) .
$$

Note that $W_{\lambda}(\omega)$ is a cylinder function. By Lemma 1,

$$
\begin{aligned}
\mathbb{E}_{\mathbb{L}_{\lambda}}\left[e^{-\beta W_{\lambda}}\right]= & \int_{X^{[0, T]}} e^{-\beta \sum_{i=1}^{n}\left(\mathcal{H}\left(\omega\left(t_{i}\right), \lambda_{i}\right)-\mathcal{H}\left(\omega\left(t_{i}\right), \lambda_{i-1}\right)\right)} \mathbb{L}_{\lambda}(d \omega) \\
= & \int_{X} d x_{0} q_{\lambda_{0}}\left(x_{0}\right) \int_{X} d x_{1} p\left(t_{0}, x_{0}, t_{1}, x_{1}, \lambda_{0}\right) \cdots \\
& \cdot \int_{X} d x_{n-1} p\left(t_{n-2}, x_{n-2}, t_{n-1}, x_{n-1}, \lambda_{n-2}\right) \\
& \cdot \int_{X} d x_{n} p\left(t_{n-1}, x_{n-1}, t_{n}, x_{n}, \lambda_{n-1}\right) e^{-\beta \sum_{i=1}^{n}\left(\mathcal{H}\left(x_{i}, \lambda_{i}\right)-\mathcal{H}\left(x_{i}, \lambda_{i-1}\right)\right)} .
\end{aligned}
$$

Assumption 2 implies that for all $s<t$, for all $y \in X$, and $\bar{\lambda} \in \Lambda$,

$$
\int_{X} d x \frac{e^{-\beta \mathcal{H}(x, \bar{\lambda})}}{e^{-\beta \mathcal{H}(y, \bar{\lambda})}} p(s, x, t, y, \bar{\lambda})=1 .
$$


Taking into account this, and changing the order of integration in (6), we obtain

$$
\begin{aligned}
\mathbb{E}_{\mathbb{L}_{\lambda}}\left[e^{-\beta W_{\lambda}}\right]= & \frac{1}{Z_{\lambda_{0}}} \int_{X} d x_{n} e^{-\beta \mathcal{H}\left(x_{n}, \lambda_{n}\right)} \\
& \cdot \int_{X} d x_{n-1} \frac{e^{-\beta \mathcal{H}\left(x_{n-1}, \lambda_{n-1}\right)}}{e^{-\beta \mathcal{H}\left(x_{n}, \lambda_{n-1}\right)}} p\left(t_{n-1}, x_{n-1}, t_{n}, x_{n}, \lambda_{n-1}\right) \cdots \\
& \cdot \int_{X} d x_{0} \frac{e^{-\beta \mathcal{H}\left(x_{0}, \lambda_{0}\right)}}{e^{-\beta \mathcal{H}\left(x_{1}, \lambda_{0}\right)}} p\left(t_{0}, x_{0}, t_{1}, x_{1}, \lambda_{0}\right) .
\end{aligned}
$$

Starting from the end, we replace each integral in (8) with 1 , which is valid by the relation (7), until we reach the very first integral (taken with respect to $x_{n}$ ), which we replace with $Z_{\lambda_{n}}$. Noticing that $\lambda_{0}=\lambda(0)$ and $\lambda_{n}=\lambda(T)$, we obtain

$$
\mathbb{E}_{\mathbb{L}_{\lambda}}\left[e^{-\beta W_{\lambda}}\right]=\frac{Z_{\lambda(T)}}{Z_{\lambda(0)}} .
$$

The theorem is proved.

\subsection{Jarzynski's identity, case $\lambda \in \mathrm{C}^{V}[0, T]$}

Theorem 2. (Jarzynski's identity: case $\left.\lambda \in \mathrm{C}^{V}[0, T]\right)$ Let $\lambda \in \mathrm{C}^{V}[0, T]$, and let the transition density function $p_{\lambda}$ satisfy Assumptions 2,3 , and 4 . Let the probability distribution $\mathbb{L}_{\lambda}$ of $\Gamma_{t}^{\lambda}$ be given by (2), and satisfy Assumption 1. In addition, let the following assumptions be fulfilled:

5. If $\lambda^{n} \in \mathcal{L}_{\text {step }}[0, T]$, and as $n \rightarrow \infty, \lambda^{n} \rightarrow \lambda$ uniformly on $[0, T]$, then $\mathbb{L}_{\lambda^{n}} \rightarrow \mathbb{L}_{\lambda}$ weakly relative to the family of bounded continuous cylinder functions;

6. The function $\mathcal{H}(\cdot, \bar{\lambda})$ is bounded and Borel measurable on $X$ for each fixed $\bar{\lambda} \in$ $\lambda([0, T])$

7. The partial derivative $\partial_{\lambda} \mathcal{H}$ exists at all points of $X \times \lambda([0, T])$, and is bounded;

8. The functions $\partial_{\lambda} \mathcal{H}(x, \cdot)$ are equicontinuous as a family of functions parametrized by $x \in X$;

9. The function $\partial_{\lambda} \mathcal{H}(\cdot, \bar{\lambda})$ is continuous for each fixed $\bar{\lambda} \in \Lambda$.

Then, the function $e^{-\beta W_{\lambda}}$ is $\mathbb{L}_{\lambda}$-integrable, and

$$
\mathbb{E}_{\mathbb{L}_{\lambda}}\left[e^{-\beta W_{\lambda}}\right]=e^{-\beta \Delta F} .
$$

Lemma 2. Let $\lambda \in V[0, T]$. Then, under Assumptions 1 and 7-9, the function $W_{\lambda}(\omega)$ given by $(5)$ is $\sigma_{\mathrm{c}}$-measurable.

Proof. Below, for an arbitrary small $\varepsilon$ we construct a $\sigma_{\mathrm{c}}$-measurable function $F: \Omega \rightarrow \mathbb{R}$ such that

$$
\sup _{\Omega}\left|F(\omega)-W_{\lambda}(\omega)\right|<\varepsilon
$$


We find a function $\lambda_{\text {step }}=\sum_{i=0}^{n-1} \lambda\left(t_{i}\right) \mathbb{I}_{\left[t_{i}, t_{i+1}\right)}+\lambda\left(t_{n}\right) \mathbb{I}_{\left\{t_{n}\right\}} \in \mathcal{L}_{\text {step }}[0, T]$ such that for all $\omega \in \Omega$, for all $t \in[0, T]$,

$$
\left|\partial_{\lambda} \mathcal{H}\left(\Gamma_{t}^{\lambda}(\omega), \lambda(t)\right)-\partial_{\lambda} \mathcal{H}\left(\Gamma_{t}^{\lambda}(\omega), \lambda_{\text {step }}(t)\right)\right|<\varepsilon .
$$

This is possible by Assumption 8. Thus, it suffices to find a measurable function $F$ verifying (10) for $W_{\lambda}$ of the form $\int_{s}^{r} \partial_{\lambda} \mathcal{H}\left(\Gamma_{t}^{\lambda}(\omega), \bar{\lambda}\right) d \lambda_{c}(t)$, where $\bar{\lambda} \in \lambda([0, T])$ is fixed, and $\lambda_{\mathrm{c}}$ is not a constant on $[s, r]$.

By Assumption 1, $\mathbb{L}_{\lambda}$-a.s., the paths of $\Gamma_{t}^{\lambda}$ are right continuous and without discontinuities of the second kind. This implies that the map $\Gamma^{\lambda}:[0, T] \times \Omega \rightarrow$ $X$ is $\left(\mathscr{B}([0, T]) \otimes \sigma_{\mathrm{c}}, \mathscr{B}(X)\right)$-measurable (where $\mathscr{B}([0, T])$ and $\mathscr{B}(X)$ are Borel $\sigma$ algebras) which follows from Ref. 17 (Chap. 2, Theorem 11). By Assumption 7 , $\partial_{\lambda} \mathcal{H}(\cdot, \cdot)$ is bounded, say by a constant $M$. We divide the ball of radius $M$ in $\mathbb{R}^{l}$ with the center in the origin into a finite number of sets $O_{i}$ whose diameter is smaller than $\frac{\varepsilon}{V_{0}^{T}\left[\lambda_{c}\right]}$, where $V_{0}^{T}\left[\lambda_{\mathrm{c}}\right]$ denotes the variation of $\lambda_{\mathrm{c}}$ on $[0, T]$. Further let $A_{i}=\partial_{\lambda} \mathcal{H}(\cdot, \bar{\lambda})^{-1}\left(O_{i}\right)$, and $\mathrm{C}_{i}=\left(\Gamma^{\lambda}\right)^{-1}\left(A_{i}\right) \subset[0, T] \times \Omega$. The sets $A_{i}$ are open by Assumption 9 . The sets $\mathrm{C}_{i}$ are $\mathscr{B}([0, T]) \otimes \sigma_{\mathrm{c}}$-measurable by the $(\mathscr{B}([0, T]) \otimes$ $\left.\sigma_{\mathrm{c}}, \mathscr{B}(X)\right)$-measurability of the map $\Gamma^{\lambda}$. Fix $x_{i} \in A_{i}$, and consider the function $\Phi(\omega, t)=\sum_{i} \partial_{\lambda} \mathcal{H}\left(x_{i}, \bar{\lambda}\right) \mathbb{I}_{\mathrm{C}_{i}}(\omega, t)$. Clearly, $\sup _{t \in[0, T], \omega \in \Omega}\left|\partial_{\lambda} \mathcal{H}(\omega(t), \bar{\lambda})-\Phi(\omega, t)\right|<$ $\frac{\varepsilon}{V_{0}^{T}\left[\lambda_{c}\right]}$. We define

$$
F(\omega)=\int_{s}^{r} \Phi(\omega, t) \lambda(d t)=\sum_{i} \partial_{\lambda} \mathcal{H}\left(x_{i}, \bar{\lambda}\right) \mu_{\lambda}\left(\left\{t:(\omega, t) \in \mathrm{C}_{i}\right\}\right),
$$

where $\mu_{\lambda}$ is the Lebesgue-Stieltjes measure on $[0, T]$ corresponding to the function $\lambda$. Fubini's theorem implies that the function $\omega \mapsto \mu_{\lambda}\left(\left\{t:(\omega, t) \in \mathrm{C}_{i}\right\}\right)$ is $\sigma_{\mathrm{c}^{-}}$ measurable. The inequality (10) is obviously satisfied.

Proof of Theorem 2. We take a sequence of partitions $\mathcal{P}_{n}=\left\{0=t_{0}^{n}<t_{1}^{n}<\right.$ $\left.\cdots<t_{n}^{n}=T\right\}$, and consider the functions

$$
\lambda^{n}(t)=\sum_{i=0}^{n-1} \lambda\left(t_{i}^{n}\right) \mathbb{I}_{\left[t_{i}^{n}, t_{i+1}^{n}\right)}(t)+\lambda(T) \mathbb{I}_{\{T\}}(t) .
$$

Clearly, $\lambda^{n} \rightrightarrows \lambda$ on $[0, T]$, and $V_{0}^{T}\left[\lambda^{n}\right]<V_{0}^{T}[\lambda]$, where $V_{0}^{T}$ is the variation on $[0, T]$. We set $\lambda_{i}^{n}=\lambda\left(t_{i}^{n}\right)$, and define the functions

$$
\varphi_{n}(\omega)=e^{-\beta \sum_{i=1}^{n}\left(\mathcal{H}\left(\omega\left(t_{i}^{n}\right), \lambda_{i}^{n}\right)-\mathcal{H}\left(\omega\left(t_{i}^{n}\right), \lambda_{i-1}^{n}\right)\right)} .
$$

By Theorem 1,

$$
\int_{\Omega} \varphi_{n}(\omega) \mathbb{L}_{\lambda^{n}}(d \omega)=\frac{Z_{\lambda(T)}}{Z_{\lambda(0)}}
$$


for all $n$. We denote $\varphi(\omega)=e^{-\beta W_{\lambda}(\omega)}$, and prove that

$$
\begin{array}{r}
\lim _{n \rightarrow \infty} \int_{\Omega}\left(\varphi_{n}(\omega)-\varphi(\omega)\right) \mathbb{L}_{\lambda^{n}}(d \omega)=0, \\
\lim _{n \rightarrow \infty} \int_{\Omega}\left(\varphi_{n}(\omega)-\varphi(\omega)\right) \mathbb{L}_{\lambda}(d \omega)=0, \\
\lim _{n \rightarrow \infty} \lim _{m \rightarrow \infty} \int_{\Omega}\left(\varphi_{n}(\omega)-\varphi(\omega)\right) \mathbb{L}_{\lambda^{m}}(d \omega)=0 .
\end{array}
$$

For this, we first replace the functions $\varphi_{n}$ in (11)-(13) with more suitable functions $\hat{\varphi}_{n}$ such that $\left(\varphi_{n}(\omega)-\hat{\varphi}_{n}(\omega)\right) \rightarrow 0$ as $n \rightarrow \infty$, uniformly on $\Omega$. We have:

$$
\begin{aligned}
\sum_{i=1}^{n} & \left(\mathcal{H}\left(\omega\left(t_{i}^{n}\right), \lambda_{i}^{n}\right)-\mathcal{H}\left(\omega\left(t_{i}^{n}\right), \lambda_{i-1}^{n}\right)\right)-\int_{0}^{T} \partial_{\lambda} \mathcal{H}\left(\omega(t), \lambda^{n}(t)\right) d \lambda^{n}(t) \\
= & \sum_{i=1}^{n} \partial_{\lambda} \mathcal{H}\left(\omega\left(t_{i}^{n}\right), \tilde{\lambda}_{i}^{n}\right)\left(\lambda_{i}^{n}-\lambda_{i-1}^{n}\right)-\sum_{i=1}^{n} \partial_{\lambda} \mathcal{H}\left(\omega\left(t_{i}^{n}\right), \lambda_{i}^{n}\right)\left(\lambda_{i}^{n}-\lambda_{i-1}^{n}\right),
\end{aligned}
$$

where in the first term in (14) we applied the mean value theorem to each summand, and chose $\tilde{\lambda}_{i}^{n} \in\left[\lambda_{i}^{n}, \lambda_{i+1}^{n}\right]$. Since by assumption $\partial_{\lambda} H(x, \cdot)$ is equicontinuous, the absolute value of the difference in (14) does not exceed $\varepsilon V_{0}^{T}[\lambda]$ where $\varepsilon$ is chosen so that $\left|\partial_{\lambda} \mathcal{H}\left(x, \bar{\lambda}_{1}\right)-\partial_{\lambda} \mathcal{H}\left(x, \bar{\lambda}_{2}\right)\right|<\varepsilon$ whenever $\left|\bar{\lambda}_{1}-\bar{\lambda}_{2}\right|<\delta$, and $\delta$ is chosen by the equicontinuity argument. The relation (14) shows that if we prove (11)-(13) with $\tilde{\varphi}_{n}(\omega)=e^{-\beta \int_{0}^{T} \partial_{\lambda} \mathcal{H}\left(\omega(t), \lambda^{n}(t)\right) d \lambda^{n}(t)}$ substituted for $\varphi_{n}$, then we prove (11)-(13). We define the functions:

$$
\begin{aligned}
\omega^{n}(t, \omega) & =\sum_{i=0}^{n-1} \omega\left(t_{i}^{n}\right) \mathbb{I}_{\left[t_{i}^{n}, t_{i+1}^{n}\right)}(t)+\omega(T) \mathbb{I}_{\{T\}}(t) ; \\
\tilde{\omega}^{n}(t, \omega) & =\omega(0) \mathbb{I}_{\{0\}}(t)+\sum_{i=1}^{n} \omega\left(t_{i}^{n}\right) \mathbb{I}_{\left(t_{i-1}^{n}, t_{i}^{n}\right]}(t) ; \\
\tilde{\lambda}^{n}(t) & =\lambda(0) \mathbb{I}_{\{0\}}(t)+\sum_{i=1}^{n} \lambda\left(t_{i}^{n}\right) \mathbb{I}_{\left(t_{i-1}^{n}, t_{i}^{n}\right]}(t) .
\end{aligned}
$$

With the help of these functions, the second term in (14) can be represented as

$$
\begin{aligned}
\int_{0}^{T} \partial_{\lambda} \mathcal{H}\left(\omega(t), \lambda^{n}(t)\right) d \lambda^{n}(t) & =\int_{0}^{T} \partial_{\lambda} \mathcal{H}\left(\omega^{n}(t, \omega), \lambda^{n}(t)\right) d \lambda^{n}(t) \\
& =\int_{0}^{T} \partial_{\lambda} \mathcal{H}\left(\tilde{\omega}^{n}(t, \omega), \tilde{\lambda}^{n}(t)\right) d \lambda(t) .
\end{aligned}
$$

By Assumption 8, we choose an $\varepsilon>0$ so that $\mid \partial_{\lambda} \mathcal{H}\left(\tilde{\omega}^{n}(t, \omega), \tilde{\lambda}^{n}(t)\right)-$ $\partial_{\lambda} \mathcal{H}\left(\tilde{\omega}^{n}(t, \omega), \lambda(t)\right) \mid<\varepsilon$ whenever $\sup _{t \in[0, T]}\left|\tilde{\lambda}^{n}(t)-\lambda(t)\right|<\delta$, and $\delta$ is chosen 
by the equicontinuity argument. This means that the relations (11), (12), and (13) are equivalent to

$$
\begin{array}{r}
\lim _{n \rightarrow \infty} \int_{\Omega}\left(\hat{\varphi}_{n}(\omega)-\varphi(\omega)\right) \mathbb{L}_{\lambda^{n}}(d \omega)=0, \\
\lim _{n \rightarrow \infty} \int_{\Omega}\left(\hat{\varphi}_{n}(\omega)-\varphi(\omega)\right) \mathbb{L}_{\lambda}(d \omega)=0, \\
\lim _{n \rightarrow \infty} \lim _{m \rightarrow \infty} \int_{\Omega}\left(\hat{\varphi}_{n}(\omega)-\varphi(\omega)\right) \mathbb{L}_{\lambda^{m}}(d \omega)=0,
\end{array}
$$

where $\hat{\varphi}_{n}(\omega)=e^{-\beta \int_{0}^{T} \partial_{\lambda} \mathcal{H}\left(\tilde{\omega}^{n}(t, \omega), \lambda(t)\right) d \lambda(t)}$, and $\tilde{\omega}^{n}(t, \omega)$ is given by (15). We show that the relations (17) hold. Since $\partial_{\lambda} \mathcal{H}(\cdot, \cdot)$ is bounded on $X \times \lambda([0, T])$ by Assumption $7, \lambda$ is a function of bounded variation on $[0, T]$, and the exponent is Lipschitz on bounded domains, for all $m$ we obtain the estimate

$$
\begin{aligned}
& \left|\int_{\Omega}\left(\hat{\varphi}_{n}(\omega)-\varphi(\omega)\right) \mathbb{L}_{\lambda^{m}}(d \omega)\right| \\
& \quad<K_{L} \int_{\Omega} \mathbb{L}_{\lambda^{m}}(d \omega) \int_{0}^{T}|\lambda|(d t)\left|\partial_{\lambda} \mathcal{H}\left(\tilde{\omega}^{n}(t, \omega), \lambda(t)\right)-\partial_{\lambda} \mathcal{H}(\omega(t), \lambda(t))\right| \\
& \quad=K_{L} \int_{0}^{T}|\lambda|(d t) \int_{\Omega} \mathbb{L}_{\lambda^{m}}(d \omega)\left|\partial_{\lambda} \mathcal{H}\left(\tilde{\omega}^{n}(t, \omega), \lambda(t)\right)-\partial_{\lambda} \mathcal{H}(\omega(t), \lambda(t))\right|,
\end{aligned}
$$

where $K_{L}$ is the Lipschitz constant for the exponent, $|\lambda|(d t)$ is the LebesgueStieltjes measure corresponding to the total variation function $|\lambda|(t)$, and Fubini's theorem has been applied to pass to the last integral. The same estimate holds for $\mathbb{L}_{\lambda}$. Namely,

$$
\begin{aligned}
& \left|\int_{\Omega}\left(\hat{\varphi}_{n}(\omega)-\varphi(\omega)\right) \mathbb{L}_{\lambda}(d \omega)\right| \\
& \quad=K_{L} \int_{0}^{T}|\lambda|(d t) \int_{\Omega} \mathbb{L}_{\lambda}(d \omega)\left|\partial_{\lambda} \mathcal{H}\left(\tilde{\omega}^{n}(t, \omega), \lambda(t)\right)-\partial_{\lambda} \mathcal{H}(\omega(t), \lambda(t))\right| .
\end{aligned}
$$

We would like to show that

$$
\begin{array}{r}
\lim _{n \rightarrow \infty} \int_{\Omega} \mathbb{L}_{\lambda^{n}}(d \omega)\left|\partial_{\lambda} \mathcal{H}\left(\tilde{\omega}^{n}(t, \omega), \lambda(t)\right)-\partial_{\lambda} \mathcal{H}(\omega(t), \lambda(t))\right|=0, \\
\lim _{n \rightarrow \infty} \int_{\Omega} \mathbb{L}_{\lambda}(d \omega)\left|\partial_{\lambda} \mathcal{H}\left(\tilde{\omega}^{n}(t, \omega), \lambda(t)\right)-\partial_{\lambda} \mathcal{H}(\omega(t), \lambda(t))\right|=0, \\
\lim _{n \rightarrow \infty} \lim _{m \rightarrow \infty} \int_{\Omega} \mathbb{L}_{\lambda^{m}}(d \omega)\left|\partial_{\lambda} \mathcal{H}\left(\tilde{\omega}^{n}(t, \omega), \lambda(t)\right)-\partial_{\lambda} \mathcal{H}(\omega(t), \lambda(t))\right|=0 .
\end{array}
$$


Let $t \in\left(t_{i-1}^{n}, t_{i}^{n}\right]$, and let $\delta_{n}=t_{i}^{n}-t$. Then $\tilde{\omega}^{n}(t, \omega)=\omega\left(t+\delta_{n}\right)$. Let us show $(20)$. We have:

$$
\begin{aligned}
\int_{\Omega} \mathbb{L}_{\lambda^{n}}(d \omega)\left|\partial_{\lambda} \mathcal{H}\left(\omega\left(t+\delta_{n}\right), \lambda(t)\right)-\partial_{\lambda} \mathcal{H}(\omega(t), \lambda(t))\right| \\
=\int_{X} q_{\lambda_{0}}\left(x_{0}\right) d x_{0} \int_{X} d x p_{\lambda^{n}}\left(t_{0}, x_{0}, t, x\right) \\
\quad \cdot \int_{X} d x_{i} p\left(t, x, t+\delta_{n}, x_{i}, \lambda(t)\right)\left|\partial_{\lambda} \mathcal{H}(x, \lambda(t))-\partial_{\lambda} \mathcal{H}\left(x_{i}, \lambda(t)\right)\right| .
\end{aligned}
$$

Note that

$$
F_{n}(x)=\int_{X} d x_{i} p\left(t, x, t+\delta_{n}, x_{i}, \lambda(t)\right)\left|\partial_{\lambda} \mathcal{H}\left(x_{i}, \lambda(t)\right)-\partial_{\lambda} \mathcal{H}(x, \lambda(t))\right|
$$

converges to zero uniformly in $x$ running over compacts in $X$ which follows from Assumption 4. Indeed,

$$
\left|F_{n}(x)\right|^{2} \leq \int_{X} d x_{i} p\left(t, x, t+\delta_{n}, x_{i}, \lambda(t)\right)\left|\partial_{\lambda} \mathcal{H}\left(x_{i}, \lambda(t)\right)-\partial_{\lambda} \mathcal{H}(x, \lambda(t))\right|^{2} .
$$

The right-hand side of this inequality converges to zero uniformly in $x \in K \subset X$, where $K$ is an arbitrary compact. This easily follows from Assumption 4 after we separate in the right-hand side the terms depending on $x$ and on $x_{i}$. Define the measures:

$$
\begin{aligned}
\mu_{\lambda^{n}}(A) & =\int_{X} q_{\lambda_{0}}\left(x_{0}\right) d x_{0} \int_{A} p_{\lambda^{n}}\left(t_{0}, x_{0}, t, x\right) d x, \\
\mu_{\lambda}(A) & =\int_{X} q_{\lambda_{0}}\left(x_{0}\right) d x_{0} \int_{A} p_{\lambda}\left(t_{0}, x_{0}, t, x\right) d x .
\end{aligned}
$$

By Assumption $5, \mathbb{L}_{\lambda^{n}}$ converges weakly to $\mathbb{L}_{\lambda}$ relative to the family of bounded continuous cylinder function, whenever $\lambda^{n} \rightrightarrows \lambda$. This implies that $\mu_{\lambda^{n}} \rightarrow \mu_{\lambda}$ weakly (relative to the family of bounded continuous functions), as $\lambda^{n} \rightrightarrows \lambda$. By Prokhorov's theorem, the family $\left\{\mu_{\lambda^{n}}, \mu_{\lambda}\right\}$ of probability measures on $X$ is tight. We fix an arbitrary $\varepsilon>0$ and find a compact $K_{\varepsilon}$ such that $\mu_{\lambda^{n}}\left(X \backslash K_{\varepsilon}\right)<\varepsilon$ for all $n$. Since $F_{n} \rightrightarrows 0$ on $K_{\varepsilon}$, and all $F_{n}$ are bounded on $X$ by a constant, say $M$, not depending on $n$, we can find an $N \in \mathbb{N}$ such that $\left|F_{n}(x)\right|<\varepsilon$ on $K_{\varepsilon}$ for all $n>N$. We obtain the estimate:

$$
\left|\int_{X} \mu_{\lambda^{n}}(d x) F_{n}(x)\right| \leq \sup _{x \in K_{\varepsilon}}\left|F_{n}(x)\right|+M \mu_{\lambda^{n}}\left(X \backslash K_{\varepsilon}\right) \leq \varepsilon+M \varepsilon .
$$

This proves (20). The relation (21) follows from the right continuity of $\omega$, Assumption 9, and Lebesgue's theorem. The relation (22) follows from Assumption 5 and (21). Application of Lebesgue's theorem to the integral (18) (taken over $[0, T]$ with 
respect to $|\lambda|(d t))$ implies that as $n \rightarrow \infty$, the integral (18) converges to zero. This implies (17), and thus (11), (12) and (13) are proved. By (11), we obtain:

$$
\begin{aligned}
0 & =\lim _{n \rightarrow \infty} \int\left(\varphi_{n} \mathbb{L}_{\lambda^{n}}-\varphi \mathbb{L}_{\lambda^{n}}\right)=\lim _{n \rightarrow \infty} \int \varphi_{n} \mathbb{L}_{\lambda^{n}}-\lim _{n \rightarrow \infty} \int \varphi \mathbb{L}_{\lambda^{n}} \\
& =\frac{Z_{\lambda(T)}}{Z_{\lambda(0)}}-\lim _{n \rightarrow \infty} \int \varphi \mathbb{L}_{\lambda^{n}} .
\end{aligned}
$$

On the other hand, (12) and (13) imply:

$$
\begin{aligned}
0 & =\lim _{n \rightarrow \infty} \lim _{m \rightarrow \infty} \int\left(\varphi_{\lambda^{n}} \mathbb{L}_{\lambda^{m}}-\varphi \mathbb{L}_{\lambda^{m}}\right) \\
& =\lim _{n \rightarrow \infty} \lim _{m \rightarrow \infty} \int \varphi_{n} \mathbb{L}_{\lambda^{m}}-\lim _{m \rightarrow \infty} \int \varphi \mathbb{L}_{\lambda^{m}} \\
& =\lim _{n \rightarrow \infty} \int \varphi_{n} \mathbb{L}_{\lambda}-\lim _{m \rightarrow \infty} \int \varphi \mathbb{L}_{\lambda^{m}}=\int \varphi \mathbb{L}_{\lambda}-\lim _{m \rightarrow \infty} \int \varphi \mathbb{L}_{\lambda^{m}} .
\end{aligned}
$$

Comparing the last two relations gives:

$$
\int_{\Omega} \varphi(\omega) \mathbb{L}_{\lambda}(d \omega)=\frac{Z_{\lambda(T)}}{Z_{\lambda(0)}}
$$

The theorem is proved for the case $\lambda \in \mathrm{C}^{V}[0, T]$.

\subsection{Jarzynski's identity for $\lambda \in V[0, T]$ and its corollaries}

Corollary 1. (Corollary of Theorem 2) Let the assumptions of Theorem 2 be fulfilled, and let $f: X \rightarrow \mathbb{R}$ be bounded Borel measurable. Then,

$$
\mathbb{E}_{\mathbb{L}_{\lambda}}\left[\left(f \circ \pi_{T}\right) e^{-\beta W_{\lambda}}\right]=\mathbb{E}_{\lambda(T)}[f] \mathbb{E}_{\mathbb{L}_{\lambda}}\left[e^{-\beta W_{\lambda}}\right],
$$

where $\pi_{t}: X^{[0, T]} \rightarrow X, \omega \mapsto \omega(t)$ is the evaluation mapping, $\mathbb{E}_{\lambda(T)}$ is the expectation relative to the measure $\frac{1}{Z_{\lambda(T)}} e^{-\beta \mathcal{H}(x, \lambda(T))} d x$.

Proof. Assuming that $\lambda \in \mathcal{L}_{\text {step }}[0, T]$, we repeat the argument of (6) and (8), while using the relation (7). Specifically, we obtain:

$$
\begin{aligned}
& \mathbb{E}_{\mathbb{L}_{\lambda}}\left[\left(f \circ \pi_{T}\right) e^{-\beta W_{\lambda}}\right] \\
&=\frac{1}{Z_{\lambda\left(t_{0}\right)}} \int_{X} e^{-\beta \mathcal{H}\left(x_{n}, \lambda\left(t_{n}\right)\right)} f\left(x_{n}\right) d x_{n} \\
& \cdot \int_{X} \frac{e^{-\beta \mathcal{H}\left(x_{n-1}, \lambda\left(t_{n-1}\right)\right)}}{e^{-\beta \mathcal{H}\left(x_{n}, \lambda\left(t_{n-1}\right)\right)} p\left(t_{n-1}, x_{n-1}, t_{n}, x_{n}, \lambda\left(t_{n-1}\right)\right) d x_{n-1} \ldots} \\
& \cdot \int_{X} \frac{e^{-\beta \mathcal{H}\left(x_{0}, \lambda\left(t_{0}\right)\right)}}{e^{-\beta \mathcal{H}\left(x_{1}, \lambda\left(t_{0}\right)\right)}} p\left(t_{0}, x_{0}, t_{1}, x_{1}, \lambda\left(t_{0}\right)\right) d x_{0} \\
&= \mathbb{E}_{\lambda(T)}[f] \frac{Z_{\lambda\left(t_{n}\right)}}{Z_{\lambda\left(t_{0}\right)}}=\mathbb{E}_{\lambda(T)}[f] \mathbb{E}_{\mathbb{L}_{\lambda}}\left[e^{-\beta W_{\lambda}}\right] .
\end{aligned}
$$


Now let $\lambda \in \mathrm{C}^{V}[0, T]$, and let the constant $M_{f}$ be such that $\sup _{x \in X}|f(x)|<M_{f}$. As in the proof of Theorem 2, we find a sequence of step functions $\lambda^{n}$ converging to $\lambda$ uniformly on $[0, T]$. The identity (23) holds for each $\lambda^{n}$. The argument of passing to the limit as $n \rightarrow \infty$ is similar to the argument used in the proof of Theorem 2. We define the function $F: \Omega \rightarrow \mathbb{R}, F(\omega)=f(\omega(T))$, and repeat the argument of Theorem 2 until the inequality (19) with the following replacements: $\varphi \leftrightarrow F \varphi, \varphi_{n} \leftrightarrow F \varphi_{n}, \tilde{\varphi}_{n} \leftrightarrow F \tilde{\varphi}_{n}, \hat{\varphi}_{n} \leftrightarrow F \hat{\varphi}_{n}$. The right-hand sides of (18) and (19) will be the same as in the proof of Theorem 2 but the Lipschitz constant $K_{L}$ will be replaced with $K_{L} M_{f}$. The part of the proof of Theorem 2 following after the inequality (19) remains unchanged until the last two arguments of passing to the limit. Those arguments now will be:

$$
\begin{aligned}
0 & =\lim _{n \rightarrow \infty} \int\left(F \varphi_{n} \mathbb{L}_{\lambda^{n}}-F \varphi \mathbb{L}_{\lambda^{n}}\right)=\lim _{n \rightarrow \infty} \int F \varphi_{n} \mathbb{L}_{\lambda^{n}}-\lim _{n \rightarrow \infty} \int F \varphi \mathbb{L}_{\lambda^{n}} \\
& =\mathbb{E}_{\lambda(T)}[f] \mathbb{E}_{\mathbb{L}_{\lambda}}\left[e^{-\beta W_{\lambda}}\right]-\lim _{n \rightarrow \infty} \int F \varphi \mathbb{L}_{\lambda^{n}} .
\end{aligned}
$$

On the other hand,

$$
\begin{aligned}
0 & =\lim _{n \rightarrow \infty} \lim _{m \rightarrow \infty} \int\left(F \varphi_{\lambda^{n}} \mathbb{L}_{\lambda^{m}}-F \varphi \mathbb{L}_{\lambda^{m}}\right) \\
& =\lim _{n \rightarrow \infty} \lim _{m \rightarrow \infty} \int F \varphi_{n} \mathbb{L}_{\lambda^{m}}-\lim _{m \rightarrow \infty} \int F \varphi \mathbb{L}_{\lambda^{m}} \\
& =\int F \varphi \mathbb{L}_{\lambda}-\lim _{m \rightarrow \infty} \int F \varphi \mathbb{L}_{\lambda^{m}} .
\end{aligned}
$$

This implies the identity (23).

Corollary 2. (Corollary of Theorem 2) Let the assumptions of Corollary 1 except Assumption 5 of Theorem 2 be fulfilled with respect to the function $\lambda \in \mathrm{C}^{V}[0, T]$. Let $a \in \Lambda$, and let $\lambda^{a}=\lambda \mathbb{I}_{[0, T)}+a \mathbb{I}_{\{T\}}$. Let us assume that

If $\lambda^{n} \in \mathcal{L}_{\text {step }}[0, T]$, and $\lambda^{n} \rightrightarrows \lambda^{a}$ on $[0, T]$, then $\mathbb{L}_{\lambda^{n}} \rightarrow \mathbb{L}_{\lambda^{a}}$ weakly relative to the family of bounded continuous cylinder functions.

Then,

$$
\mathbb{E}_{\mathbb{L}_{\lambda^{a}}}\left[\left(f \circ \pi_{T}\right) e^{-\beta W_{\lambda}}\right]=\mathbb{E}_{\lambda(T)}[f] \frac{Z_{\lambda(T)}}{Z_{\lambda(0)}} .
$$

Proof. If $\lambda \in \mathcal{L}_{\text {step }}[0, T]$, then $\lambda^{a}=\sum_{i=1}^{n-1} \lambda_{i} \mathbb{I}_{\left[t_{i}, t_{i+1}\right)}+a \mathbb{I}_{\{T\}}$. The representation (4) shows that $\mathbb{E}_{\mathbb{L}_{\lambda^{a}}}\left[\left(f \circ \pi_{T}\right) e^{-\beta W_{\lambda}}\right]$ is the same for the functions $\lambda^{a}$ with different a. Hence,

$$
\mathbb{E}_{\mathbb{L}_{\lambda^{a}}}\left[\left(f \circ \pi_{T}\right) e^{-\beta W_{\lambda}}\right]=\mathbb{E}_{\lambda(T)}[f] \frac{Z_{\lambda(T)}}{Z_{\lambda(0)}} .
$$


Now let $\lambda \in \mathrm{C}^{V}[0, T]$. Note that in the relations (18) and (19) we integrate with respect to $|\lambda|(d t)$ where $|\lambda|$ is continuous. Hence, by Lebesgue's theorem, the investigation of convergence at the point $T$ in (20)-(22) is not necessary to prove the convergence in (18) and (19). Finally we note that by assumption, and by the relations (19) and (21), with $\lambda^{a}$ substituted for $\lambda$, for the first term in the identity (24) we obtain:

$$
\lim _{n \rightarrow \infty} \lim _{m \rightarrow \infty} \int F \varphi_{n} \mathbb{L}_{\lambda^{m}}=\int F \varphi \mathbb{L}_{\lambda^{a}} .
$$

This proves (25).

Let $[s, t] \subset[0, T]$ be an arbitrary subinterval, $X^{[s, t]}$ be the space of all functions on $[s, t]$. We introduce further notation: Let $\mathrm{C}^{V}[s, t]$ be the space of continuous functions of bounded variation on $[s, t], \mathcal{L}_{\text {step }}[s, t]$ be the space of right continuous step functions on $[s, t]$ taking a finite number of values. Below, on $X^{[s, t]}$ we define the distributions $\mathbb{L}_{\lambda ; s, t}^{x}$ and $\mathbb{L}_{\lambda ; s, t}, \lambda \in V[0, T], x \in X$. Let $s<\tau_{1}<\cdots<\tau_{k-1}<t$ be a partition of $[s, t], f: X^{k+1} \rightarrow \mathbb{R}$ be bounded and measurable. The finitedimensional distributions of $\mathbb{L}_{\lambda ; s, t}^{x}$ we define by the formula:

$$
\begin{aligned}
\int_{X^{[s, t]}} & f\left(\omega(s), \omega\left(\tau_{1}\right), \ldots, \omega\left(\tau_{k-1}\right), \omega(t)\right) \mathbb{L}_{\lambda ; s, t}^{x}(d \omega) \\
= & \left.\int_{X} d x_{1} p_{\lambda}\left(s, x, \tau_{1}, x_{1}\right)\right) \cdots \int_{X} d x_{k} p_{\lambda}\left(\tau_{k-1}, x_{k-1}, t, x_{k}\right) f\left(x, x_{1}, \ldots, x_{k}\right) .
\end{aligned}
$$

The finite-dimensional distributions of $\mathbb{L}_{\lambda ; s, t}$ we define by:

$$
\mathbb{L}_{\lambda ; s, t}[f]=\mathbb{E}_{\lambda(s)}\left[\mathbb{L}_{\lambda ; s, t}[f]\right] .
$$

We extend the measures $\mathbb{L}_{\lambda ; s, t}^{x}$ and $\mathbb{L}_{\lambda ; s, t}$ to $\sigma_{\mathrm{c}}\left(X^{[s, t]}\right)$ by Kolmogorov's extension theorem. We assume that $\sigma_{\mathrm{c}}\left(X^{[s, t]}\right)$ is augmented with all subsets of $\mathbb{L}_{\lambda ; s, t}$-null sets.

Theorem 3. (Jarzynski's identity, case $\lambda \in V[0, T]$ ) Let $\lambda \in V[0, T]$, and let $\left\{t_{1}<\cdots<t_{k}=T\right\}$ be the set of its discontinuity points. Further let the transition density function $p_{\lambda}$ of the Markov process $\Gamma_{t}^{\lambda}$ satisfy Assumptions 2, 3 and 4, and the probability distribution $\mathbb{L}_{\lambda}$ be given by (2), and satisfy Assumption 1. Let Assumptions 6, 7,8 and 9 of Theorem 2 be fulfilled. Additionally, we assume that

10. If $\lambda^{m} \in \mathcal{L}_{\text {step }}[0, T]$, and $\lambda^{m} \rightrightarrows \lambda$ on $[0, T]$, as $m \rightarrow \infty$, then for each $i, 0 \leq i<$ $n, \mathbb{L}_{\lambda^{m} ; t_{i}, t_{i+1}} \rightarrow \mathbb{L}_{\lambda ; t_{i}, t_{i+1}}$ weakly relative to the family of bounded continuous cylinder functions on $X^{\left[t_{i}, t_{i+1}\right]}$;

11. For all bounded and continuous functions $f: X^{\left[t_{i}, t_{i+1}\right]} \rightarrow \mathbb{R}$, the function $X \rightarrow$ $\mathbb{R}, x \mapsto \mathbb{L}_{\lambda ; t_{i}, t_{i+1}}^{x}[f]$ is Borel measurable.

Then, the function $e^{-\beta W_{\lambda}}$ is $\mathbb{L}_{\lambda}$-integrable, and

$$
\mathbb{E}_{\mathbb{L}_{\lambda}}\left[e^{-\beta W_{\lambda}}\right]=e^{-\beta \Delta F} .
$$

The continuity of functions $X^{\left[t_{i}, t_{i+1}\right]} \rightarrow \mathbb{R}$ is understood with respect to the topology of pointwise convergence. 
Proof of Theorem 3, case $\boldsymbol{\lambda} \in \boldsymbol{V}[\mathbf{0}, \boldsymbol{T}]$. Let $\lambda=\lambda_{\mathrm{c}}+\lambda_{\text {step }}$, where $\lambda_{\mathrm{c}} \in \mathrm{C}^{V}[0, T]$, and $\lambda_{\text {step }}=\sum_{i=0}^{n-1} \lambda_{i} \mathbb{I}_{\left[t_{i}, t_{i+1}\right)}+\lambda_{n} \mathbb{I}_{\{T\}} \in \mathcal{L}_{\text {step }}[0, T]$. On each interval $\left[t_{i-1}, t_{i}\right]$ we define the function $\omega_{i}=\left.\omega\right|_{\left[t_{i-1}, t_{i}\right]}$, and identify each $\omega \in X^{[0, T]}$ with the sequence $\left(\omega_{1}, \ldots, \omega_{n}\right)$. Since $\Gamma_{t}^{\lambda}$ is a Markov process, we obtain:

$$
\begin{aligned}
\int_{X[0, T]} & e^{-\beta W_{\lambda}(\omega)} \mathbb{L}_{\lambda}(d \omega) \\
= & \int_{X} d x_{0} q_{\lambda\left(t_{0}\right)}\left(x_{0}\right) \int_{X^{\left[t_{0}, t_{1}\right]}} \mathbb{L}_{\lambda ; t_{0}, t_{1}}^{x_{0}}\left(d \omega_{1}\right) \\
& \cdot \int_{X^{\left[t_{1}, t_{2}\right]}} \mathbb{L}_{\lambda ; t_{1}, t_{2}}^{\omega_{1}\left(t_{1}\right)}\left(d \omega_{2}\right) \cdots \\
& \cdot \int_{X^{\left[t_{n-1}, t_{n}\right]}} \mathbb{L}_{\lambda ; t_{n-1}, t_{n}}^{\omega_{n-1}\left(t_{n-1}\right)}\left(d \omega_{n}\right) e^{-\beta W_{\lambda}\left(\omega_{1}, \ldots, \omega_{n}\right)},
\end{aligned}
$$

where

$$
\begin{aligned}
W_{\lambda}\left(\omega_{1}, \ldots, \omega_{n}\right)= & \sum_{i=1}^{n} \int_{t_{i-1}}^{t_{i}} \partial_{\lambda} \mathcal{H}\left(\omega_{i}(t), \lambda(t)\right) d \lambda_{\mathrm{c}}(t) \\
& +\sum_{i=1}^{n}\left(\mathcal{H}\left(\omega_{i}\left(t_{i}\right), \lambda\left(t_{i}\right)\right)-\mathcal{H}\left(\omega_{i}\left(t_{i}\right), \lambda\left(t_{i}-0\right)\right)\right) .
\end{aligned}
$$

On each interval $\left[t_{i-1}, t_{i}\right]$, we define continuous functions $\lambda_{(i)}(t)=\lambda(t), t \in\left[t_{i-1}, t_{i}\right)$, and $\lambda_{(i)}\left(t_{i}\right)=\lambda\left(t_{i}-0\right), 1 \leq i \leq n$. Let

$$
W_{\lambda_{(i)}}\left(\omega_{i}\right)=\int_{t_{i-1}}^{t_{i}} \partial_{\lambda} \mathcal{H}\left(\omega_{i}(t), \lambda(t)\right) d \lambda_{(i)}(t)
$$

We obtain:

$$
\begin{aligned}
\int_{X[0, T]} & e^{-\beta W_{\lambda}(\omega)} \mathbb{L}_{\lambda}(d \omega) \\
= & \int_{X} d x_{0} q_{\lambda\left(t_{0}\right)}\left(x_{0}\right) \\
& \cdot \int_{X^{\left[t_{0}, t_{1}\right]}} e^{-\beta W_{\lambda_{(1)}}\left(\omega_{1}\right)} e^{-\beta\left(\mathcal{H}\left(\omega_{1}\left(t_{1}\right), \lambda\left(t_{1}\right)\right)-\mathcal{H}\left(\omega_{1}\left(t_{1}\right), \lambda\left(t_{1}-0\right)\right)\right)} \mathbb{L}_{\lambda ; t_{0}, t_{1}}^{x_{0}}\left(d \omega_{1}\right) \\
& \cdot \int_{X^{\left[t_{1}, t_{2}\right]}} e^{-\beta W_{\lambda_{(2)}}\left(\omega_{2}\right)} e^{-\beta\left(\mathcal{H}\left(\omega_{2}\left(t_{2}\right), \lambda\left(t_{2}\right)\right)-\mathcal{H}\left(\omega_{2}\left(t_{2}\right), \lambda\left(t_{2}-0\right)\right)\right)} \mathbb{L}_{\lambda ; t_{1}, t_{2}}^{\omega_{1}\left(t_{1}\right)}\left(d \omega_{2}\right) \cdots \\
& \cdot \int_{X^{\left[t_{n-1}, t_{n}\right]}} e^{-\beta W_{\lambda_{(n)}}\left(\omega_{n}\right)} e^{-\beta\left(\mathcal{H}\left(\omega_{n}\left(t_{n}\right), \lambda\left(t_{n}\right)\right)-\mathcal{H}\left(\omega_{n}\left(t_{n}\right), \lambda\left(t_{n}-0\right)\right)\right)} \mathbb{L}_{\lambda ; t_{n-1}, t_{n}}^{\omega_{n-1}\left(t_{n-1}\right)}\left(d \omega_{n}\right) .
\end{aligned}
$$


Let $\mathbb{E}_{\bar{\lambda}}$ denote the expectation relative to the measure $q_{\bar{\lambda}}(x) d x$. We can rewrite the above relation in terms of expectations:

$$
\begin{aligned}
\mathbb{E}_{\mathbb{L}_{\lambda}}\left[e^{-\beta W_{\lambda}}\right] \\
=\mathbb{E}_{\lambda\left(t_{0}\right)}\left[\mathbb { E } _ { \mathbb { L } _ { \lambda _ { ; } ; t _ { 0 } , t _ { 1 } } } \left[e^{-\beta W_{\lambda_{(1)}}\left(\omega_{1}\right)} e^{-\beta\left(\mathcal{H}\left(\omega_{1}\left(t_{1}\right), \lambda\left(t_{1}\right)\right)-\mathcal{H}\left(\omega_{1}\left(t_{1}\right), \lambda\left(t_{1}-0\right)\right)\right)} \mathbb{E}_{\mathbb{L}_{\lambda ; t_{1}, t_{2}}^{\omega_{1}\left(t_{1}\right)}}\right.\right. \\
\left.\left.\quad \cdot\left[\cdots \mathbb{E}_{\mathbb{L}_{\lambda ; t_{n-1}, t_{n}}^{\omega_{n-1}\left(t_{n-1}\right)}}\left[e^{-\beta W_{\left.\lambda_{(n)}\right)}\left(\omega_{n}\right)} e^{-\beta\left(\mathcal{H}\left(\omega_{n}\left(t_{n}\right), \lambda\left(t_{n}\right)\right)-\mathcal{H}\left(\omega_{n}\left(t_{n}\right), \lambda\left(t_{n}-0\right)\right)\right)}\right] \cdots\right]\right]\right] .
\end{aligned}
$$

Note that in (27) we always deal with a function of the form:

$$
X^{\left[t_{i-1}, t_{i}\right]} \rightarrow \mathbb{R}, \omega \mapsto e^{-\beta W_{\lambda_{(i)}}(\omega)} f\left(\omega\left(t_{i}\right)\right),
$$

where $f$ is bounded and Borel measurable by Assumptions 9 and 11. Applying Corollary 2, we obtain:

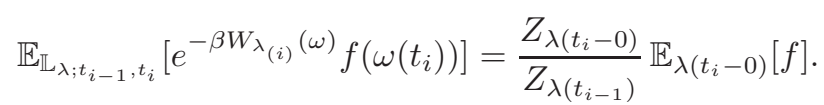

Also, note that the following relation holds:

$$
\begin{aligned}
& \mathbb{E}_{\lambda\left(t_{i}-0\right)}\left[e^{-\beta\left(\mathcal{H}\left(\bullet, \lambda\left(t_{i}\right)\right)-\mathcal{H}\left(\bullet, \lambda\left(t_{i}-0\right)\right)\right.} \mathbb{E}_{\mathbb{L}_{\lambda_{;} t_{i}, t_{i+1}}}[F]\right] \\
& \quad=\frac{Z_{\lambda\left(t_{i}\right)}}{Z_{\lambda\left(t_{i}-0\right)}} \mathbb{E}_{\lambda\left(t_{i}\right)}\left[\mathbb{E}_{\mathbb{L}_{\lambda ; t_{i}, t_{i+1}}}[F]\right]=\frac{Z_{\lambda\left(t_{i}\right)}}{Z_{\lambda\left(t_{i}-0\right)}} \mathbb{E}_{\mathbb{L}_{\lambda ; t_{i}, t_{i+1}}}[F] .
\end{aligned}
$$

Applying the identities (30) and (29) to (27), we obtain:

$$
\mathbb{E}_{\mathbb{L}_{\lambda}}\left[e^{-\beta W_{\lambda}}\right]=\frac{Z_{\lambda\left(t_{1}-0\right)}}{Z_{\lambda\left(t_{0}\right)}} \frac{Z_{\lambda\left(t_{1}\right)}}{Z_{\lambda\left(t_{1}-0\right)}} \cdots \frac{Z_{\lambda\left(t_{n}-0\right)}}{Z_{\lambda\left(t_{n-1}\right)}} \frac{Z_{\lambda\left(t_{n}\right)}}{Z_{\lambda\left(t_{n}-0\right)}}=\frac{Z_{\lambda(T)}}{Z_{\lambda(0)}} .
$$

Corollary 3. (Corollary of Theorem 3) Let the assumptions of Theorem 3 be fulfilled, and let $f: X \rightarrow \mathbb{R}$ be bounded and Borel measurable. Then,

$$
\mathbb{E}_{\mathbb{L}_{\lambda}}\left[\left(f \circ \pi_{T}\right) e^{-\beta W_{\lambda}}\right]=\mathbb{E}_{\lambda(T)}[f] \mathbb{E}_{\mathbb{L}_{\lambda}}\left[e^{-\beta W_{\lambda}}\right] .
$$

Proof. We repeat the argument that we used in the proof of Theorem 3 to obtain (27) in connection to the expression $\mathbb{E}_{\mathbb{L}_{\lambda}}\left[\left(f \circ \pi_{T}\right) e^{-\beta W_{\lambda}}\right]$. Instead of the very last expectation in (27), we obtain

$$
\mathbb{E}_{\mathbb{L}_{\lambda ; t_{n-1}, t_{n}}^{\omega_{n-1}\left(t_{n-1}\right)}}\left[e^{-\beta W_{\lambda_{(n)}}\left(\omega_{n}\right)} f\left(\omega_{n}\left(t_{n}\right)\right) e^{-\beta\left(\mathcal{H}\left(\omega_{n}\left(t_{n}\right), \lambda\left(t_{n}\right)\right)-\mathcal{H}\left(\omega_{n}\left(t_{n}\right), \lambda\left(t_{n}-0\right)\right)\right)}\right] .
$$


Applying Corollary 2, we obtain:

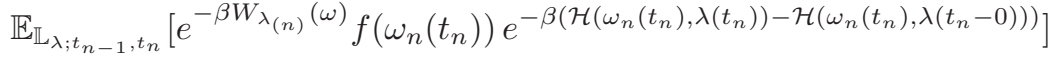

$$
\begin{aligned}
& =\frac{Z_{\lambda\left(t_{n}-0\right)}}{Z_{\lambda\left(t_{n-1}\right)}} \mathbb{E}_{\lambda\left(t_{n}-0\right)}\left[e^{-\beta\left(\mathcal{H}\left(\cdot, \lambda\left(t_{n}\right)\right)-\mathcal{H}\left(\cdot, \lambda\left(t_{n}-0\right)\right)\right)} f\right] \\
& =\frac{Z_{\lambda\left(t_{n}-0\right)}}{Z_{\lambda\left(t_{n-1}\right)}} \frac{Z_{\lambda\left(t_{n}\right)}}{Z_{\lambda\left(t_{n}-0\right)}} \mathbb{E}_{\lambda\left(t_{n}\right)}[f] .
\end{aligned}
$$

Instead of the multiplier $\frac{Z_{\lambda\left(t_{n}-0\right)}}{Z_{\lambda\left(t_{n}-1\right)}} \frac{Z_{\lambda\left(t_{n}\right)}}{Z_{\lambda\left(t_{n}-0\right)}}$ in the relation (31) we obtain $\frac{Z_{\lambda\left(t_{n}-0\right)}}{Z_{\lambda\left(t_{n-1}\right)}} \frac{Z_{\lambda\left(t_{n}\right)}}{Z_{\lambda\left(t_{n}-0\right)}} \mathbb{E}_{\lambda\left(t_{n}\right)}[f]$ according to (33). This gives (32).

\section{Bochkov-Kuzovlev's Identity}

Here we give a rigorous mathematical proof of the identity announced in Refs. 4-7 where the authors used a different definition of work to that in Refs. 1 and 2. The difference between the two definitions of work was analyzed in Ref. 3, and it was found that

$$
W(\omega)-W_{0}(\omega)=\mathcal{H}(\omega(T), \lambda(T))-\mathcal{H}(\omega(T), \lambda(0)),
$$

where $W_{0}$ is the work in the Bochkov-Kuzovlev sense. We use this equality as the definition of the new work $W_{0}$, and will prove the following theorem:

Theorem 4. Let the assumptions of Theorem 3 be fulfilled. Then,

$$
\mathbb{E}_{\mathbb{L}_{\lambda}}\left[e^{-\beta W_{0}}\right]=1 .
$$

Moreover, the identities (34) and (26) are equivalent.

Proof. Applying Corollary 3 and the identity (26) we obtain:

$$
\begin{aligned}
& \mathbb{E}_{\mathbb{L}_{\lambda}}\left[e^{-\beta W_{0}}\right]=\mathbb{E}_{\mathbb{L}_{\lambda}}\left[e^{-\beta W}\right] \mathbb{E}_{\lambda(T)}\left[e^{-\beta(\mathcal{H}(\omega(T), \lambda(T))-\mathcal{H}(\omega(T), \lambda(0)))}\right] \\
& =\mathbb{E}_{\mathbb{L}_{\lambda}}\left[e^{-\beta W}\right] \frac{Z_{\lambda(0)}}{Z_{\lambda(T)}}=\frac{Z_{\lambda(T)}}{Z_{\lambda(0)}} \frac{Z_{\lambda(0)}}{Z_{\lambda(T)}}=1 .
\end{aligned}
$$

This relation also shows that the identities (34) and (26) are equivalent.

\section{Acknowledgments}

The author thanks C. Dellago for attracting her attention to Jarzynski's identity, and O. G. Smolyanov for useful discussions. This work was supported by the research grant of the Erwin Schrödinger Institute for Mathematical Physics (Vienna), by the Austrian Science Fund (FWF) under START-prize-grant Y328, and by the Portuguese Foundation for Science and Technology (FCT) under the project PTDC/MAT/69635/06. 


\section{References}

1. C. Jarzynski, Nonequilibrium equality for free energy differences, Phys. Rev. Lett. 78 2690 (1997) 2690-2693.

2. C. Jarzynski, Equilibrium free energy differences from nonequilibrium measurements: A master-equation approach, Phys. Rev. E. 56 (1997) 5018-5035.

3. C. Jarzynski, Comparison of far-from-equilibrium work relations, Comptes Rend. Phys. 8 (2007) 495-506.

4. G. N. Bochkov and Yu. E. Kuzovlev, General theory of thermal fluctuations in nonlinear systems, Zh. Eksp. Teor. Fiz. 72 (1977) 238-243 [JETP 45 (1977) 125-130].

5. G. N. Bochkov and Yu. E. Kuzovlev, Fluctuation-dissipation relations for nonequilibrium processes in open systems, Zh. Eksp. Teor. Fiz. 76 (1979) 1071-1079 [JETP 49 (1979) 543-551].

6. G. N. Bochkov and Yu. E. Kuzovlev, Nonlinear fluctuation-dissipation relations and stochastic models in nonequilibrium thermodynamics, I. Generalized fluctuationdissipation theorem, Physica 106A (1981) 443-479.

7. G. N. Bochkov and Yu. E. Kuzovlev, Nonlinear fluctuation-dissipation relations and stochastic models in nonequilibrium thermodynamics, II. Kinetic potential and variational principles for nonlinear irreversible processes, Physica 106A (1981) 480.

8. G. E. Crooks, Path-ensemble averages in systems driven far from equilibrium, Phys. Rev. E. 61 (2000) 2361-2366.

9. G. E. Crooks, Nonequilibrium measurements of free energy. Differences for microscopically reversible Markovian systems, J. Statist. Phys. 90 (1998) 1481-1487.

10. G. E. Crooks, Entropy production fluctuation theorem and the nonequilibrium work relation for free energy differences, Phys. Rev. E 60 (1999) 2721-2726.

11. C. Dellago and E. Schöll-Paschinger, A proof of Jarzynski's nonequilibrium work theorem for dynamical systems that conserve the canonical distribution, J. Chem. Phys. 125 (2006) 054105.

12. C. Dellago, P. L. Geissler, W. Lechner and H. Oberhofer, Equilibrium free energies from fast-switching simulations with large time steps, J. Chem. Phys. 124 (2006) 044113.

13. E. B. Dynkin, Markov Processes (Academic Press, 1965).

14. G. Hummer and A. Szabo, Free energy reconstruction from nonequilibrium singlemolecule pulling experiments, Proc. Nat. Acad. Sci. 98 (2001) 3658.

15. G. Hummer and A. Szabo, Free energy surfaces from single-molecule force spectroscopy, Acc. Chem. Res. 38 (2005) 504-513.

16. J. Liphardt et al., Equilibrium information from nonequilibrium measurements in an experimental test of Jarzynski's equality, Science 296 (2002) 1832.

17. N. I. Portenko, A. V. Skorokhod and B. M. Shurenkov, Markov processes, Itogi nauki i techniki. Sovremennye problemy matematiki, Fund. Talnye Napravleniya 46 (1989) 5-248 (in Russian). 